DOI: 10.17816/byusu20210179-84

УДК 314.114:004.94

Работа выполнена при поддержке гранта РФФИ, проект № 18-47-860016

\author{
С. П. Семенов, В. В. Славский, М. В. Куркина, \\ А. О. Ташкин, О. В. Самарина, А. А. Финогенов \\ КОМПЬЮТЕРНЫЕ МАТЕМАТИЧЕСКИЕ МОДЕЛИ \\ СОЦИАЛЬНО-ЭКОНОМИЧЕСКИХ СИСТЕМ \\ С ИСПОЛЬЗОВАНИЕМ ГИС-ТЕХНОЛОГИЙ
}

В статье описань результаты пяти лет исследований в области разработки и создания компьютерных математических моделей социально-экономических систем с использованием ГИС-технологий в рамках двух грантов РФФИ, которыми руководил В. В. Славский. Первая часть описывает результаты по разработке и созданию интерактивной сочиильно-ориентированной геоинформационный системы, предназначенной для получения информации об объектах соичальной инфраструктуры, направленной на удовлетворение потребностей маломобильных групп граждан и людей с ограниченными возможностями. Вторая часть описывает результаты по разработке и созданию интерактивной агентно-ориентированной динамической модели сочиильно-экономических прочессов (транспортных, производственных, демографических, и пр.), с использованием ГИС-технологий на примере г. Ханть-Мансийска.

Ключевые слова: город, динамика, сочиальный, инфраструктура, ОСИ, карта, доступность, модели, ГИС, ХМАО, Ханть-Мансийск.

S. P. Semenov, V. V. Slavsky, M. V. Kurkina, A. O. Tashkin, O. V. Samarina, A. A. Finogenov

\title{
COMPUTER MATHEMATICAL MODELS OF SOCIO-ECONOMIC SYSTEMS USING GIS TECHNOLOGIES
}

The article describes the results of five years of research in the development and creation of computer mathematical models of socio-economic systems using GIS technologies within the framework of two RFBR grants, which were directed by V.V. Slavsky. The first part describes the results of the development and creation of an interactive socially-oriented geographic information system designed to obtain information about social infrastructure facilities aimed at meeting the needs of people with limited mobility and people with disabilities. The second part describes the results of the development and creation of an interactive agent-based dynamic model of socio-economic processes (transport, production, demographic, etc.), using GIS technologies on the example of KhantyMansiysk.

Key words: city, dynamics, social, infrastructure, OSI, map, accessibility, models, GIS, KhantyMansiysk.

Данная статья посвящена памяти нашего коллеги и товарища, известного ученого профессора В. В. Славского, который почти 20 лет проработал на кафедре высшей математики Югорского Государственного Университета.

Виктор Владимир Славский, обладая высоким уровнем знаний и невероятной энциклопедичностью, смог обеспечить руководство в решении научных задач из предметных областей широкого круга. Благодаря своему профессионализму и лидерским качествам Виктор Влади- 
мирович выступал в коллективе в роли учителя и наставника. Ему сопутствовали такие личные качества, как отзывчивость, деликатность, надежность, ответственность и тонкое чувство юмора, что делало его настоящим и верным другом, незаменимым в любом научном и жизненном вопросе.

Под руководством профессора В. В. Славского в последнее десятилетие выполнялись научные гранты РФФИ, а именно: № 15-41-00092 «Фолксономический подход в разработке социально-ориентированных геоинформационных систем» и № 18-47-860016 «Компьютерное моделирование динамики социально-экономической системы ресурсодобывающего региона севера России с использованием теории роста, агентного подхода и ГИС-технологий», в работе над которыми принимали участие и авторы данной статьи.

Работа над грантами сопровождалась серией публикаций в различных международных изданиях, 12 из которых входят в реестр базы данных SCOPUS, 17 публикаций изданы в сборниках, включенных в перечень ВАК, 36 публикаций в изданиях, входящих в РИНЦ [1-8]. Помимо этого, были изданы 3 методических пособия, принималось участие в 43 международных и российских конференциях.

В рамках исследовательской работы над грантом РФИИ № 15-41-00092 «Фолксономический подход в разработке социально-ориентированных геоинформационных систем» был получен ряд результатов, наиболее значимые из которых следующие:

- Произведен анализ социально-ориентированных Российских и зарубежных разработок, направленных на удовлетворение информационных потребностей, а также и социальноориентированных ГИС-продуктов. Установлены возможности и задачи, реализуемые данными продуктами, определен их функционал и средства разработки. Выявлены наиболее удачные решения, применительно к задаче удовлетворения информационных потребностей маломобильных групп граждан [1].

- Произведен анализ информационной потребности маломобильных граждан и людей с ограниченными возможностями. Выработаны решения по наиболее выгодной организации информационных ресурсов, направленных на удовлетворение информационной потребности определенной категории граждан.

- Произведен анализ средств разработки и выявление наиболее оптимальных подходов к созданию проблемно-ориентированных социальных информационных ресурсов [2].

- Спроектировано и реализовано программно-техническое решение для обеспечения возможности сотрудничества группы людей с целью накопления, хранения, анализа и обмена социально-ориентированными данными.

- Спроектирована и реализована интерактивная социально-ориентированная геоинформационная система, предназначенная для получения информации об объектах социальной инфраструктуры, направленная на удовлетворение потребностей маломобильных групп граждан и людей с ограниченными возможностями. На рисунке 1 представлен скриншот созданной геоинформационной системы для МГН, опубликованный в сети Интернет по адресу geowheel.ru [3].

- Изучены подходы классификации данных. Выявлены недостатки и преимущества методов классификации применительно к задачам сортировки и упорядочивания социальноориентированных данных. Экспериментальным способом выявлены наиболее адекватные методы классификации данных с целью проведения анализа формальных признаков метода классификации. Онтология представлена как знаковая система, следующим образом: $O N=\left\langle C, R, L, P_{C}, P_{L C}, P_{L R},\right\rangle$, где $C=\left\{c_{1}, \ldots, c_{m}\right\}$ - множество понятий в онтологии; $R=\left\{r_{1}, \ldots, r_{m}\right\}-$ множество бинарных отношений $r_{i}\left(c_{x}, c_{y}\right)$ между понятиями; $L=$ $\left\{l_{1}, \ldots, l_{m}\right\}$ - множество лексических меток (словарь онтологии).

- Исследованы возможности комбинирования фолксономического и таксономического подходов классификации данных с целью выявления и объединения наилучших качеств 
каждого из подходов. Выработаны алгоритмические решения по объединению различных методов классификацию с целью более точного описания свойств и характеристик объектов предметной области. Фолксономическую структуру данных можно трактовать в виде множеств, обладающих свойствами: $O=\left\{O_{i}\right\}_{i=1}^{I}$-множество объектов; $P=$ $\left\{p_{j}\right\}_{j=1}^{J}$; множество признаков объектов $U=\left\{u_{n}\right\}_{n=1}^{N}$ - множество пользователей; первые два множества $O, P$ генерируются третьим множеством $U ; O, P, U=\left\{o_{i}, p_{j}, u_{n}\right\}_{i=1, \mathrm{j}=1, \mathrm{n}=1}^{I J N}$

- Проанализированы особенности классификации слабосвязанных социально-ориентированных данных. Выявлены наиболее оптимальные подходы упорядочивания и сортировки контекстной и семантической информации применительно к социально-ориентированным данным.

- Исследованы возможности динамического обновления свойств объектов предметной области. Выявлены методы и алгоритмы динамического обновления свойств и характеристик объектов для увеличения релевантности объекта классификации.

- Проанализированы методы публичного использования информационных социально-ориентированных ресурсов. Исследованы возможности классификации одного объекта предметной области сразу несколькими аутентичными логически несвязными свойствами.

- Исследованы возможности формально-контекстного анализа с целью организации классификации социально-ориентированных и пространственных данных и выявления наиболее оптимальных семантических решений. Для обозначения отношения между множествами объектов и признаков $(O, P)$ введено множество $\left.Y=\left\{<o_{i}, p_{j}\right\rangle\right\}_{i=1, j=1}^{I J}$, которое содержит информацию о том, каким набором из множества признаков наделен каждый объект из множества ОСИ, $Y \subseteq O \times P$, отношение $<o_{i}, p_{j}>$ означает, что объект $o_{i}$ обладает признаком $p_{j}$. Формальный контекст выражен как $\mathbb{K}=(O, P, Y)$ и представлен в виде бинарной матрицы.

- Изучены возможности установления связей между объектами предметной области, исследованы инструменты и алгоритмы поиска связей между данными. Реализован оригинальный подход поиска связности и близости социально-ориентированных данных с целью организации классификации данных.

- Произведен анализ методов и возможностей построения математических моделей организации классификации социально-ориентированных данных с помощью инструментов формально-контекстного анализа. На основе двух множеств (объекты и их признаки) и известного бинарного отношения между ними, используя операторы Галуа, можно получить формальные понятия. Для произвольных $A \subseteq P$ и $B \subseteq O$ определены операторы Галуа: $\quad A^{\prime}=\{p \in P \mid \forall o \in A:(o Y p)\} ; B^{\prime}=\{o \in O \mid \forall p \in B:(o Y p)\} . A^{\prime}$ - это множество признаков, которыми наделены все объекты, представленные в множестве $A$. $B^{\prime}$ - это множество признаков, которыми наделены все объекты, представленные в множестве $B$. Формальное понятие $(A, B)$ включает в себя множество объектов $A \subseteq O$ и множество признаков $B \subseteq P$, удовлетворяющих условию $B^{\prime}=A$ и $A^{\prime}=B$.

- Построены новые алгоритмы, направленные на выявление наиболее оптимальных информационно-аналитических решений посредством анализа единиц естественного языка и речи в многообразии семантической информации социально-ориентированных данных.

- Спроектирован и реализован программный модуль, осуществляющий классификацию данных на основании разработанных подходов и алгоритмов классификации данных. Произведена интеграция модуля в разработанную ГИС-систему. На рисунке 2 представлена структурно-функциональная схема геоинформационной системы для МГН в которой отражены элементы и модули ГИС. 
- Произведен анализ эффективности исследования применительно к задаче удовлетворения информационной потребности маломобильных групп граждан и людей с ограниченными возможностями $[4,5]$.

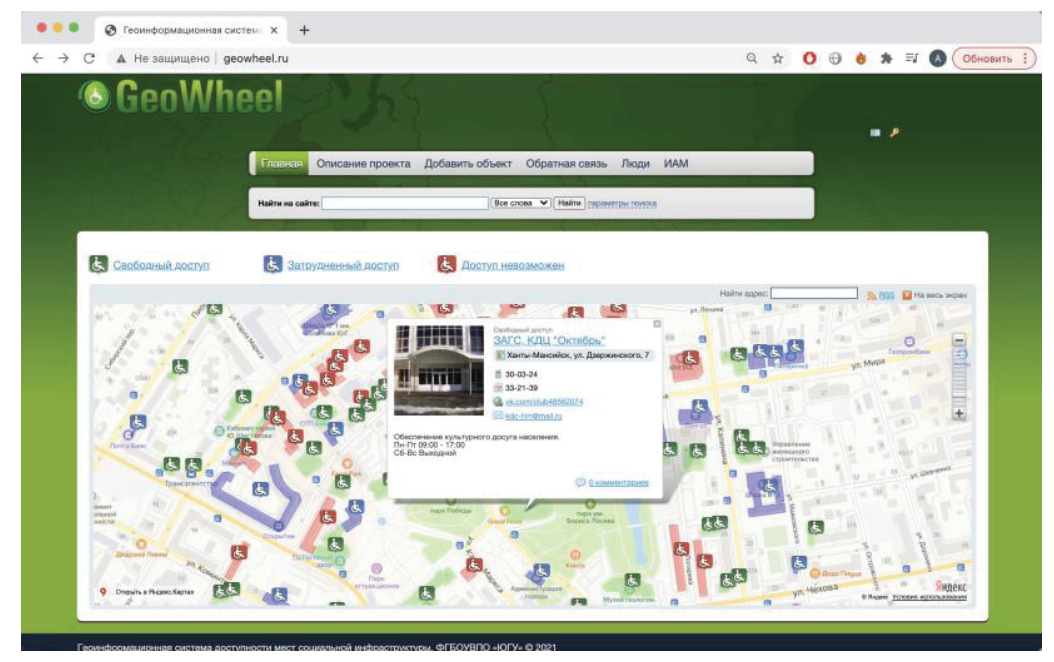

Рисунок 1 - Скриншот геоинформационной системы geowheel.ru

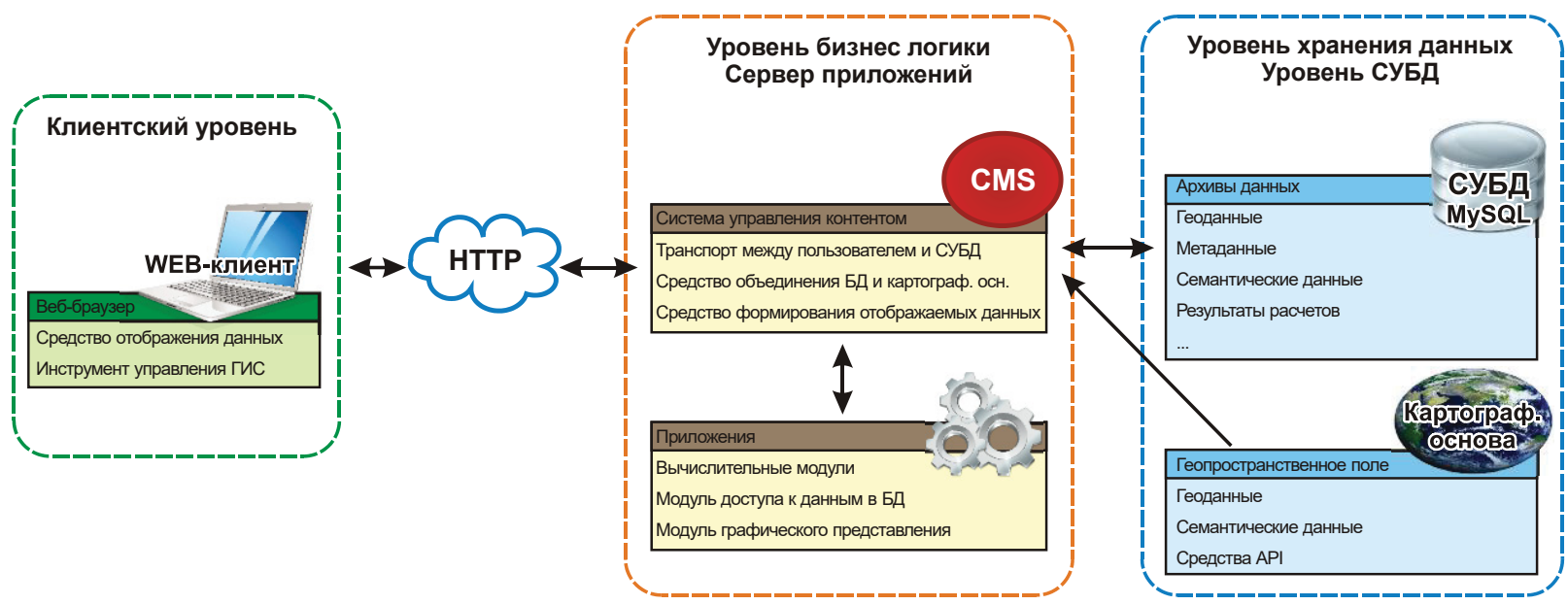

Рисунок 2 - Структурно-функциональная схема системы

В рамках исследовательской работы над грантом РФФИИ № 18-47-860016 «Компьютерное моделирование динамики социально-экономической системы ресурсодобывающего региона севера России с использованием теории роста, агентного подхода и ГИС-технологий» был получен ряд результатов, наиболее значимые из них следующие:

- Изучены современные подходы и возможности многоагентного моделирования социально-экономических систем и средств распределенного программирования с использованием ГИС-технологий.

- Исследованы технологии мобильных программных агентов для решения задач имитационного моделирования динамических процессов в сложных социально-экономических системах.

- Разработаны концептуальные агентно-ориентированные модели социально-экономических процессов (транспортных, производственных, демографических, и пр.), направленных на изучение влияния экономических параметров на качество жизни ресурсодобывающего региона севера России на примере одного города. Описаны алгоритмы, концептуальные и логические схемы модели, входные и выходные параметры [6].

- Разработана интерактивная агентно-ориентированная динамическая модель с использованием ГИС-технологий города северного региона России. На примере г. Ханты-Мансийска выполнено создание модели с помощью компьютерных средств имитационного 
моделирования [6]. С созданной моделью проведен ряд экспериментов, выявлены различные сценарии работы модели, определены критические показатели, построены графики и диаграммы на основе полученных в ходе экспериментов данных. На рисунке 3 представлена структурно-функциональная схема разработанной модели.

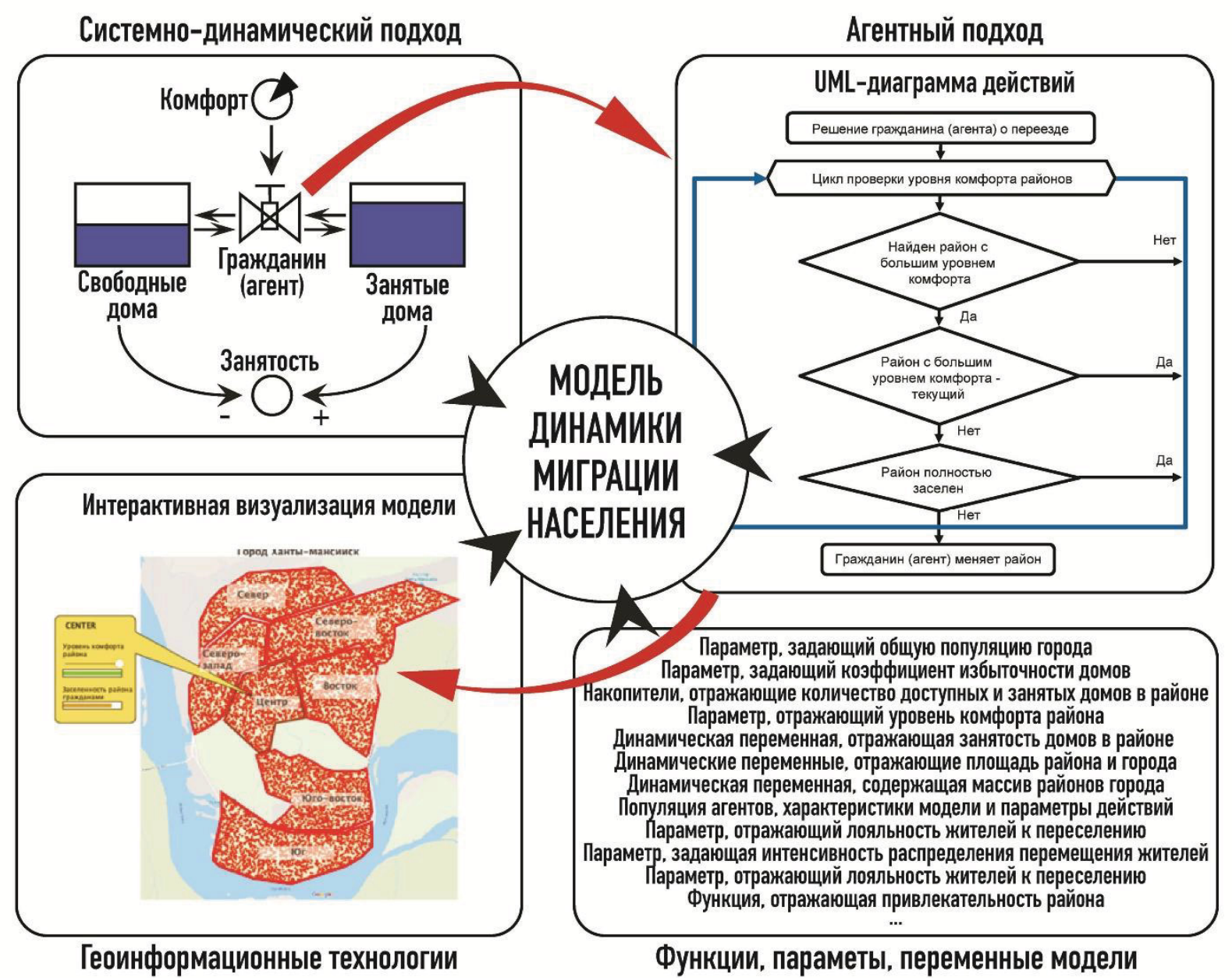

Рисунок 3 - Концептуальная схема модели

Уход из жизни В. В. Славского - руководителя проекта, произошел во время работы по гранту. Научное исследование и работа в рамках поставленных грантом задач продолжены членами коллектива и авторами данной статьи.

\section{Литература}

1. Mathematical model of social infrastructure based on the theory of formal concept analysis (FCA) / S. P. Semenov, V. V. Slavskii, A. O. Tashkin, A. S. Tyakunov// International journal of engineering \& technical research. - 2017. - Vol. 7, Issue 8. - P. 2291.

2. Математическая модель объектов социальной инфраструктуры на основе теории анализа формальных понятий (АФП) / С. П. Семенов, В. В. Славский, А. О. Ташкин, А. С. Тякунов. - Текст : непосредственный // Инновации в жизнь : международный научный журнал. 2017. - № 2 (21). - С. 175-186.

3. Семенов, С. П. Анализ информационных ресурсов, направленных на удовлетворение информационных потребностей людей с ограниченными возможностями / С. П. Семенов, В. В. Славский, А. О. Ташкин. - Текст : непосредственный // Вестник НГУ Серия: Информационные технологии. - 2015. - Т. 13, Вып. № 2. - С. 84-102. 
4. Семенов, С. П. Разработка имитационной модели геоинформационной системы для маломобильных групп населения / С. П. Семенов, В. В. Славский, А. О. Ташкин. - Текст : непосредственный // Вестник Югорского Государственного университета. - 2017. - № 4. - С. 78-85.

5. Семенов, С. П. Оценка эффективности ГИС для маломобильных групп населения / С. П. Семенов, А. О. Ташкин. - Текст : непосредственный // Труды конференции МАК: «Математики Алтайскому краю» : сборник трудов всероссийской конференции по математике с международным участием. - Барнаул : Издательство Алтайского университета, 2018. -С. 121-125.

6. Агентно-ориентированные имитационные модели для реальных городских процессов / С. М. Пронь, С. П. Семенов, А. О. Ташкин, Е. В. Токарева. - Текст : непосредственный // Труды конференции МАК: «Математики - Алтайскому краю» : сборник трудов всероссийской конференции по математике с международным участием. - Барнаул : Издательство Алтайского университета, 2019. - С. 169-173.

7. Самарина, О. В. Применение интегральных топографических характеристик в решении задач обработки данных дистанционного зондирования / О. В. Самарина, С. П. Семенов, В. В. Славский. - Текст : непосредственный // Математические заметки СВФУ. - 2020. - Т. 27, № 1. - C. 41-50.

8. Ташкин, А. О. Социально-ориентированные геоинформационные системы, модели и методы реализации / С. П. Семенов, В. В. Славский, А. О. Ташкин. - Текст : непосредственный // Материалы III Всероссийской научно-практической конференции АУ «Югорский НИИ информационных технологий». - Ханты-Мансийск : ТехноКом, 2017. - С. 25-32. 